Artículo

\title{
Óptimos económicos de fertilizante en la producción de maíz blanco en Valles Altos
}

\author{
Manuel Vargas-Salgado ${ }^{1 \S}$ \\ Marcos Portillo-Vázquez ${ }^{1}$ \\ José de Jesús Brambila-Paz ${ }^{2}$ \\ Miguel Ángel Martínez-Damián ${ }^{2}$ \\ Sergio Ernesto Medina-Cuellar ${ }^{3}$
}

${ }^{1}$ Universidad Autónoma Chapingo. Carretera México-Texcoco km 38.5, Chapingo, Texcoco, Estado de México. CP. 56230. Tel. 55 17266142. (mportillo49@yahoo.com.mx). ${ }^{2}$ Colegio de Postgraduados. Carretera México-Texcoco km 36.5, Montecillo, Texcoco, México. CP. 56230. (jbrambilaa@colpos.mx; ange101@colpos.mx). ${ }^{3}$ Campus Irapuato-Salamanca-Universidad de Guanajuato. Carretera SalamancaValle de Santiago km 3.5+1.8, Salamanca, Guanajuato. CP. 36885. (se.medina@ugto.mx).

Autor para correspondencia: mvargass@ chapingo.mx.

\section{Resumen}

La producción de maíz se realiza en la mayoría de las áreas agrícolas en el país, México cuenta con cerca de 64 razas de maíces seleccionados en miles de años por grupos indígenas y mestizos además de híbridos y variedades mejoradas. Las tecnologías de producción empleadas por los productores son diversas, van desde las tradicionales pasando por las intermedias hasta las más modernas que usan paquetes tecnológicos avanzados. Estas últimas aplican altas dosis de fertilizantes y densidades de siembra elevadas, con procesos mecanizados para obtener altos rendimientos por hectárea; mientras que otros agricultores que aplican menores dosis de fertilización, con rendimientos más bajos alcanzan mejores ganancias. El presente trabajo se propone identificar en experimentos de campo, la dosis óptima económica de fertilizante (F) para que los productores logren la máxima ganancia. El experimento se realizó en el Campo Agrícola Experimental de la Universidad Autónoma Chapingo empleando un diseño experimental completamente al azar con cuatro repeticiones, durante el ciclo primavera-verano de 2019. La función de producción cuadrática de maíz blanco estimada permitió calcular la dosis óptima económica o de máxima ganancia de $\mathrm{F}$ ( $848 \mathrm{~kg} \mathrm{ha}^{-1}$ ), tomando en cuenta los precios de mercado del maíz y del fertilizante; también la dosis de máximo rendimiento (1 $893 \mathrm{~kg} \mathrm{ha}^{-1}$ ). Los resultados obtenidos servirán de criterio económico para la toma de decisiones de producción tanto de productores pequeños con escasos recursos, como de medianos y de grandes productores con mayor cantidad de recursos.

Palabras clave: función de producción, máxima ganancia, máximo rendimiento.

Recibido: julio de 2021

Aceptado: octubre de 2021 


\section{Introducción}

La producción de maíz blanco se realiza en casi todas las regiones geográficas y zonas rurales de México en una gran diversidad de ambientes: húmedos, secos, fríos y calientes; también se cultiva desde el nivel del mar hasta cerca de los $3400 \mathrm{msnm}$, con una diversidad de prácticas agrícolas y de usos de más de 80 etnias indígenas y de muchos pueblos mestizos. En México existen alrededor de 64 razas de maíces (CONABIO, 2021) resultado de un proceso de domesticación, selección y evolución bajo cultivo realizada sobre todo por los grupos indígenas desde hace 8000 años y más recientemente también por los grupos mestizos (Wellhausen et al., 1951; Kato et al., 2009; Biodiversidad, 2020; SAGARPA, 2012).

Es el grano por excelencia por lo que representó $88.3 \%$ de la producción nacional de granos básicos en el año agrícola 2020 (SADER-SIAP, 2020). El cual se produce bajo diferentes sistemas de producción y con distintas tecnologías empleadas que involucran desde pequeñas unidades de producción (UP) de autoconsumo familiar que dedican más del 80 por ciento de su producción a su alimentación, UP semi comerciales medianas que venden más de $63 \%$ del grano, UP medianas de autoconsumo ganadero que destinan más de 83 por ciento del maíz que cultivan para alimentar a los animales que crían y las UP comerciales grandes que vende más de $96 \%$ de su producción a la industria tortillera nacional y la que elabora alimentos para consumo animal, féculas, aceite vegetal y almidones, entre otros productos (Yunes y López, 2021).

No obstante, la gran riqueza de biodiversidad y de las condiciones naturales, culturales y experiencia milenaria de su producción por grupos étnicos, no se produce todo el maíz que la sociedad mexicana requiere para satisfacer sus necesidades alimenticias. En 2019 se importó una cantidad equivalente al 38\% del consumo aparente nacional y representó 59\% de la producción nacional (SEADER-SIAP, 2020), aunque la mayor parte fue de maíz amarillo, materia prima necesaria para elaborar alimentos para consumo pecuario y para la agroindustria; lo cual refleja una fuerte dependencia del abasto exterior.

Derivado de los sistemas de producción, las dosis de fertilizante pueden ser muy variadas, mientras algunos productores de maíz blanco en México aplican altas dosis de fertilizantes y densidades de siembra elevadas, con procesos muy mecanizados para obtener altos rendimientos por hectárea, para obtener buenas ganancias, por otro lado, hay agricultores con menos recursos, que aplican inferiores dosis de fertilización y por ende, rendimientos inferiores pero logran mayores ganancias por hectárea debido a que usan más eficientemente este insumo. La hipótesis es que los primeros estén usando sobredosis de insumos que los lleve a obtener menores beneficios económicos por hectárea.

Esto constituye un problema económico, sobre todo para los que comercializan el grano y para los cuales se puede evidenciar una solución económica. Los productores que aplican dosis inferiores a la óptima económica podrían elevar sus beneficios si emplearan la dosis óptima en una superficie menor de acuerdo con su disponibilidad de recursos. El objetivo que se persigue en esta investigación es identificar las dosis óptimas económicas de fertilizante en experimentos de campo, lo cual permitirá a los productores de maíz emplear sus recursos escasos de manera más eficiente para lograr la máxima ganancia de la inversión en fertilizantes. 


\section{Metodología}

Para abordar este estudio primero se analiza el uso de funciones matemáticas que representan empíricamente la ley de los rendimientos decrecientes al utilizar funciones matemáticas principalmente de tipo exponencial, polinomios de tercer y segundo grado, entre otras (Martínez, 1972; Beattie y Taylor, 1993; Nicholson, 2002). Para evidenciarlo, como primer punto se establece la relación que existe entre los nutrientes y el rendimiento (Martínez, 1972; Jauregui y Sain, 1992; Portillo, 2015), la cual se expresa de forma matemática de la siguiente manera: Sea la dosis de los nutrientes (insumos) $\mathrm{N}, \mathrm{P}, \mathrm{K}, \ldots$, aplicados al suelo por unidad de superficie, denotados por las letras $n, p, k, \ldots, \mathrm{y} Y$ el rendimiento del cultivo por unidad de superficie. $\mathrm{Y}=\mathrm{f}(\mathrm{n}, \mathrm{p}, \mathrm{k}),(1)$. Donde $\mathrm{f}$ puede establecerse por una función cúbica, cuadrática, exponencial, etc. Se denotará a la relación (1) como 'función de producción'.

Desde el punto de vista económico la aplicación de $\mathrm{N}, \mathrm{P}, \mathrm{K}, \ldots$, tiene un costo que está determinado por la relación: $\mathrm{CT}=\mathrm{g}(\mathrm{n}, \mathrm{p}, \mathrm{k}, \ldots)$ (2). Donde: $\mathrm{CT}$ es el costo total de aplicación de los nutrientes por unidad de superficie, el cual es una función de las dosis de $n, p, k, \ldots$, se denotará a la relación (2) como 'función de costo total'. Una de las funciones de mayor uso en la práctica, es una función de la forma: $\mathrm{CT}=\mathrm{CF}+\mathrm{P}_{\mathrm{N}} n+\mathrm{P}_{\mathrm{P}} p+\mathrm{P}_{\mathrm{K}} k+\ldots$, (3). Donde: $\mathrm{CF}$ representa los costos fijos de aplicación y los símbolos $\mathrm{P}_{\mathrm{N}}, \mathrm{P}_{\mathrm{P}}, \mathrm{P}_{\mathrm{K}}, \ldots$, los precios unitarios de mercado de los nutrientes $\mathrm{N}, \mathrm{P}$, $\mathrm{K}, \ldots$, respectivamente. El ingreso total del productor viene dado por $\mathrm{P}_{\mathrm{Y}} \mathrm{Y}$. Donde: $\mathrm{P}_{\mathrm{Y}}$ es el precio unitario de mercado del producto.

Definiendo el óptimo económico como la cantidad de nutriente que genera el rendimiento o la producción de máxima ganancia o beneficio económico, dicha cantidad de insumo se obtiene, igualando el valor del producto marginal del insumo ( $\mathrm{VPMgI}=\mathrm{PMgI} * \mathrm{PYY}_{\mathrm{Y}}$ ) al precio del insumo $\mathrm{P}_{\mathrm{l}}=\mathrm{P}_{\mathrm{N}}, \mathrm{P}_{\mathrm{P}}, \mathrm{P}_{\mathrm{K}}, \ldots$, lo cual es equivalente a igualar el producto marginal del insumo (recurso), $(\mathrm{PMgI}=\partial \mathrm{Y} / \partial \mathrm{I})$ a la relación de precios del nutriente con el precio del producto $\left(\mathrm{P}_{\mathrm{I}} / \mathrm{P}_{\mathrm{Y}}\right)$, como se plantea en la ecuación (4). Matemáticamente, el problema se circunscribe a encontrar el máximo del producto $\mathrm{PYY}_{\mathrm{Y}}$ sujeto a la restricción (3) suponiendo una función de costo lineal. Formando la siguiente función de Lagrange: $\mathrm{L}=\mathrm{P}_{\mathrm{Y}} \mathrm{Y}+\lambda\left(\mathrm{CT}-\mathrm{CF}-\mathrm{P}_{\mathrm{N}} n-\mathrm{P}_{\mathrm{P}} p-\mathrm{P}_{\mathrm{K}} k-\ldots\right)$

Derivando L sucesivamente con respecto a $n, p, k, \ldots$, e igualando las igualdades a la relación de precio insumo/producto, se obtiene: $\frac{\partial \mathrm{L}}{\partial \mathrm{n}}=\frac{\mathrm{P}_{\mathrm{N}}}{\mathrm{P}_{\mathrm{Y}}}, \frac{\partial \mathrm{L}}{\partial \mathrm{p}}=\frac{\mathrm{P}_{\mathrm{P}}}{\mathrm{P}_{\mathrm{Y}}}(4), \frac{\partial \mathrm{L}}{\partial \mathrm{k}}=\frac{\mathrm{P}_{\mathrm{K}}}{\mathrm{P}_{\mathrm{Y}}}$. El sistema de ecuaciones (4) se resuelve simultáneamente. Los valores que se obtengan de $n, p, k, \ldots$, deben satisfacer al sistema y son las dosis óptimas de los recursos. Cuando se consideran los CF de la aplicación de los fertilizantes, puede suceder que el valor del incremento en el rendimiento no sea suficiente para solventar los CT de aplicación. Las aplicaciones de dosis bajas pueden ser insuficientes para pagar los $\mathrm{CF}$, aun cuando cubran los costos variables $\mathrm{P}_{\mathrm{N}} n+\mathrm{P}_{\mathrm{P}} p+\mathrm{P}_{\mathrm{K}} k+\ldots$, de la ecuación (3).

Del planteamiento anterior se deduce que debe existir un valor mínimo de $n, p, k, \ldots$, cuyas aplicaciones inferiores a estas dosis mínimas producirán pérdidas en lugar de la ganancia deseada. Basados en Pesek y Heady (1958) citados en (Martínez, 1972) se consideren como dosis mínimas de aplicación las cantidades de insumos que hacen máximas las ganancias netas por unidad monetaria. Considerando que la ganancia total es la diferencia: $\pi=\mathrm{P}_{\mathrm{Y}} \mathrm{Y}-\mathrm{CT}$; de donde la ganancia neta por unidad monetaria que se gasta se denota como $G$ y se define como sigue: $G=\left(P_{Y} Y\right.$ $\mathrm{CT}) / \mathrm{CT}$. Las dosis de $\mathrm{N}, \mathrm{P}, \mathrm{K}, \ldots$, para las cuales $\mathrm{G}$ es un máximo se calculan resolviendo simultáneamente el sistema: $\frac{\partial \mathrm{L}}{\partial \mathrm{n}}=0 ; \frac{\partial \mathrm{L}}{\partial \mathrm{p}}=0(5) ; \frac{\partial \mathrm{L}}{\partial \mathrm{k}}=0$. 
Nótese que CT en el sistema anterior no es una constante, si no que CT es una función de las dosis $n, p, k, \ldots$ Las cantidades que se obtienen al resolver el sistema (5), establecen las cantidades mínimas que se requieren para obtener las mayores ganancias, es decir, el agricultor debe fertilizar con las cantidades mínimas recomendadas la superficie que alcance estas condiciones y el resto que no puede alcanzar el nivel mínimo de fertilización se debe dejar sin sembrar (Martínez, 1972; Portillo, 2015), toda vez que otra combinación distinta de dosis y superficie reducirá las ganancias para una cantidad determinada de fertilizante.

Esto se explica porque la respuesta clásica de los cultivos a las adiciones sucesivas de fertilizantes sigue la Ley de Rendimientos 'marginales” decrecientes'. '... a dosis bajas de los nutrientes, incrementos en las dosis producen incrementos mayores en los rendimientos, e inversamente a dosis altas de los nutrientes, incrementos en las dosis producen incrementos menores en los rendimientos' (Martínez, 1972). Por lo que se utilizan funciones matemáticas que representan empíricamente la ley de los rendimientos decrecientes.

Sin embargo, los polinomios de segundo grado permiten una representación muy razonable de las respuestas de un cultivo a las aplicaciones de nutrientes. Este polinomio de segundo grado permite calcular el óptimo económico y el óptimo técnico. Este polinomio de segundo grado permite calcular el óptimo económico y el óptimo técnico por los efectos lineal y cuadrático que se observan entre los nutrientes y el rendimiento de un cultivo. Para el caso de 1 y 2 dos insumos se tienen las ecuaciones siguientes: $N: Y=\beta_{0}+\beta_{1 n}+\beta_{2 n^{2}}(6)$. N, P: Y $=\beta_{0}+\beta_{1 n}+\beta_{2 p}+\beta_{3 n^{2}}+$ $\beta_{4 \mathrm{p}^{2}}+\beta_{5 \mathrm{np}}(7)$.

El sistema de ecuaciones (4) se reduce sencillamente a la siguiente ecuación para el caso de un nutriente o de una fórmula balanceada de fertilizante $(\mathrm{F}): \beta_{1}+2 \beta_{2 \mathrm{n}}=\frac{\mathrm{P}_{\mathrm{N}}}{\mathrm{P}_{\mathrm{Y}}}$. Donde: $\mathrm{n}^{*}=$ $\frac{1}{2 \beta_{2}}\left(\frac{\mathrm{P}_{\mathrm{N}}}{\mathrm{P}_{\mathrm{Y}}}-\beta_{1}\right)(8)$. Donde: $\mathrm{n}^{*}$ o f* es la dosis óptima de $\mathrm{N}$ o de $\mathrm{F}$, respectivamente.

\section{Establecimiento del experimento}

\section{Ubicación del área de estudio}

El experimento se estableció el 27 de mayo de 2019. Se utilizaron dos variedades de maíz (Estrella y Celeste) a las cuales se les aplicó diferentes dosis de fertilización con la finalidad de evaluar posteriormente el rendimiento de grano. El experimento se desarrolló en el Campo Agrícola Experimental 'El Ranchito', en el lote X-11, perteneciente a la Universidad Autónoma Chapingo, Texcoco de Mora, Estado de México. El lote se ubica geográficamente entre los paralelos $19^{\circ} 24^{\prime}$ y $19^{\circ} 33^{\prime}$ de latitud norte, los meridianos $98^{\circ} 38^{\prime}$ y $99^{\circ} 02^{\prime}$ de longitud oeste.

\section{El análisis de suelo}

Previo al establecimiento del cultivo se realizó un muestreo de suelo para determinar su fertilidad; la cual se establecio a partir de 15 submuestras de manera aleatoria y representativa del terreno, y finalmente formar una muestra compuesta y enviarla al laboratorio de análisis químico Nutrelab, mediante los métodos establecidos en la Norma Oficial Mexicana NOM-021- SEMARNAT-2000. 


\section{Diseño de tratamientos}

El cultivo se desarrolló bajo condiciones de temporal con riegos de auxilio a los ochenta y seís días, noventa y ocho y ciento nueve días después de la siembra debido a la sequía que se presentó durante el ciclo del cultivo. Los tratamientos se dispusieron en un diseño completamente al azar (DCA) con cuatro repeticiones cada una. Cada lote o unidad experimental midió $8.8 \mathrm{~m}$ de ancho por $20 \mathrm{~m}$ de largo dando una superficie de $176 \mathrm{~m}^{2}$ y una densidad de siembra de 1056 plantas, equivalente a 60000 plantas ha ${ }^{-1}$.

Durante el desarrollo del cultivo se realizaron las prácticas agrícolas de fertilización, deshierbe, escarda, aporque y riegos de auxilio hasta su maduración. Se cosecharon en promedio 5 mazorcas en un metro lineal, en competencia completa, en cada uno de los tratamientos habiendo transcurrido 163 días desde su siembra. El grano fue secado hasta alcanzar una humedad promedio de 14\%, se desgranó y se pesó en una balanza analítica; para estimar el rendimiento promedio por hectárea de maíz. Los datos fueron capturados, ordenados y sistematizados para ser analizados en un análisis de varianza (Anova) con el paquete Sistem Analysis Statistical (SAS) versión 9, para probar la igualdad de tratamientos con el fin de aceptar o rechazar la hipótesis nula. Para cada variable evaluada se efectuó un análisis de varianza, pruebas de comparación de medias utilizando la prueba de Tukey $(\alpha=0.05)$ y la diferencia mínima significativa honesta (DMSH). Se usó el análisis de regresión para estimar la función de producción y se interpretaron y discutieron los resultados.

\section{Resultados y discusión}

Los resultados del análisis de suelo encontraron bajo contenido de materia orgánica y de nitrógeno inorgánico $(\mathrm{N})$, mientras que, cantidades en exceso de fósforo $\left(\mathrm{P}_{2} \mathrm{O}_{5}\right)$ y de potasio $\left(\mathrm{K}_{2} \mathrm{O}\right)$. En contenido de micronutrientes, hubo deficiencia de $\mathrm{Cu}$, nivel bajo de $\mathrm{Fe}$ y $\mathrm{Zn}$, medio de $\mathrm{CaO}$, alto de Mn y exceso de Mg y de B. El CIC también fue bajo y medio el pH (Cuadro 1). Tomando en cuenta esta información y los requerimientos del cultivo de maíz para producir un rendimiento promedio esperado de $10 \mathrm{t} \mathrm{ha}^{-1}$, se elaboró la fórmula balanceada de fertilizante $(\mathrm{F})$ con los contenidos siguientes: $23 \%$ de $\mathrm{N}, 0 \%$ de $\mathrm{P}_{2} \mathrm{O}_{5}, 26.53 \%$ de $\mathrm{K}_{2} \mathrm{O}, 17.73 \%$ de $\mathrm{CaO}$ más la mezcla de micronutrientes antes mencionados.

Cuadro 1. Resultado del análisis de suelo y la fórmula de fertilización.

\begin{tabular}{cccccccc}
\hline \multirow{2}{*}{ Nutrientes } & $\begin{array}{c}\text { Contenido } \\
\left(\mathrm{mg} \mathrm{kg}^{-1}\right)\end{array}$ & $\begin{array}{c}\text { Clasificación } \\
\text { nutrimental }\end{array}$ & $\begin{array}{c}\text { Recomendaciones de } \\
\text { fertilizante }\left(\mathrm{kg} \mathrm{ha}^{-1}\right)\end{array}$ & \multicolumn{4}{c}{$\begin{array}{c}\text { Fórmula de fertilización } \\
\left(\mathrm{kg} \mathrm{ha}^{-1}\right)\end{array}$} \\
\hline $\begin{array}{c}\text { Materia orgánica } \\
(\%)\end{array}$ & 1.41 & Bajo & 14516.7 & - & - & - & - \\
$\begin{array}{c}\text { Nitrógeno } \\
\text { inorgánico }\end{array}$ & 14 & Bajo & 161.8 & 92 & 138 & 276 & 414 \\
Fósforo $\left(\mathrm{P}_{2} \mathrm{O}_{5}\right)$ & 49.59 & Exceso & 3.4 & 0 & 0 & 0 & 0 \\
Potasio $\left(\mathrm{K}_{2} \mathrm{O}\right)$ & 293.74 & Exceso & 186.7 & 106 & 159 & 318 & 476 \\
Calcio $\left(\mathrm{C}_{\mathrm{a}} \mathrm{O}\right)$ & 1410.96 & Medio & 125 & 71 & 106 & 213 & 319 \\
Magnesio $(\mathrm{Mg})$ & 631.9 & Exceso & 0 & 0 & 0 & 0 & 0 \\
\hline
\end{tabular}




\begin{tabular}{cccccccc}
\hline \multirow{2}{*}{ Nutrientes } & $\begin{array}{c}\text { Contenido } \\
\left(\mathrm{mg} \mathrm{kg}^{-1}\right)\end{array}$ & $\begin{array}{c}\text { Clasificación } \\
\text { nutrimental }\end{array}$ & $\begin{array}{c}\text { Recomendaciones de } \\
\text { fertilizante }\left(\mathrm{kg} \mathrm{ha}^{-1}\right)\end{array}$ & \multicolumn{4}{c}{$\begin{array}{c}\text { Fórmula de fertilización } \\
\left(\mathrm{kg} \mathrm{ha}^{-1}\right)\end{array}$} \\
\hline Hierro (Fe) & 10.26 & Bajo & 2.18 & 0.56 & 1.08 & 2.18 & 3.27 \\
Manganeso $(\mathrm{Mn})$ & 47.42 & Alto & 0 & 0 & 0 & 0 & 0 \\
Zinc $(\mathrm{Zn})$ & 0.85 & Bajo & 5.3 & 1.59 & 2.65 & 5.30 & 7.95 \\
Cobre $(\mathrm{Cu})$ & 0.5 & Deficiente & 3.4 & 1.02 & 1.7 & 3.4 & 5.1 \\
Boro $(\mathrm{B})$ & 0.72 & Exceso & 1.14 & 0.34 & 0.57 & 1.14 & 1.71 \\
CIC (me $\left.100 \mathrm{~g}^{-1}\right)$ & 12 & Bajo & - & - & - & - & - \\
Azufrar $(\mathrm{pH})$ & 5.85 & Medio & - & - & - & - & - \\
\hline
\end{tabular}

$\mathrm{CIC}=$ capacidad de intercambio catiónico. $\mathrm{Me}=$ miliequivalentes por $100 \mathrm{~g}$. Análisis de suelo realizado por el laboratorio Nutrelab.

Una vez determinada la fórmula requerida de fertilizante se estableció el diseño experimental el cual fue de tratamientos bifactorial $2 \times 5$. El primer factor consistió en dos variedades de maíz (Estrella y Celeste). El segundo factor en cinco dosis de fertilización (T0= testigo absoluto; $\mathrm{T} 1=$ dosis del productor; T2=1/2 de la dosis óptima; T3= dosis óptima y T4:1+1/2 de la dosis óptima). El T0 consistió en no aplicar ningún tipo de fertilizante al suelo o a la planta, el T1 fue la que aplican los productores de la región $\left(400 \mathrm{~kg} \mathrm{ha}^{-1} \mathrm{de} \mathrm{F}\right)$ y la dosis óptima se realizó con base en el análisis de suelo la cual fue de $1200 \mathrm{~kg} \mathrm{ha}^{-1}$ (con 276N-0P-318K-213CaO + micronutrientes) y sirvió de punto de referencia para los otros tratamientos.

\section{Análisis de varianza}

En el Cuadro 2, se observó que la variedad Estrella presentó los mayores rendimientos estadísticos. De igual manera, el T4 generó los valores más altos en esta variable mientras que en los tratamientos T1, T2 y T3 no hubo diferencia significativa. Por otro lado, el testigo, tal como se esperaba, produjo los rendimientos más bajos de grano. Estos resultados concuerdan con los presentados por Martínez-Gutiérrez et al. (2018) determinaron las características agronómicas de híbridos blancos en cinco ambientes diferentes, la respuesta mejor, así como definir el efecto de la interacción genotipo $\times$ ambiente sobre el rendimiento y obtuvieron una productividad media de $12 \mathrm{t} \mathrm{ha}^{-1}$.

Por otra parte, Zamudio et al. (2015), evaluaron seis híbridos en tres sitios del Estado de México, fertilizados con $250 \mathrm{~kg} \mathrm{ha}^{-1}$ de $\mathrm{N}$ y cuatro tratamientos de fertilización foliar y obtuvieron un rendimiento promedio de $12.07 \mathrm{t} \mathrm{ha}^{-1}$ de grano de maíz. Aguilar-Carpio et al. (2015) analizaron el rendimiento y rentabilidad de maíz en función del genotipo, biofertilizante y nitrógeno en Iguala, Guerreo, México en 2011 y encontraron que la materia seca (MS) y el rendimiento en grano (RG) más alto se logró con la combinación de H-562, biofertilizante y $160 \mathrm{~kg} \mathrm{~N} \mathrm{ha}^{-1}(30000$ y $9243 \mathrm{~kg}$ $\mathrm{ha}^{-1}$, respectivamente) y el más bajo correspondió al tratamiento VS-535, con biofertilizante (Bio) y sin nitrógeno (17 030 y $3760 \mathrm{~kg} \mathrm{ha}^{-1}$, respectivamente). 
Cuadro 2. Anova y comparación de medias de rendimiento en $\mathbf{t ~ h a}^{-1}$ de dos variedades de maíz bajo cinco dosis de fertilización.

\begin{tabular}{ccccc}
\hline FV & - & F & - & Pr> F \\
\hline & & Rendimiento & \\
Variedad & - & 26.01 & - & $<0.0001$ \\
Dosis & - & 18.02 & - & $<0.0001$ \\
CV (\%) & - & - & 5.9 & - \\
& - & E & - & $12.77 \mathrm{a}$ \\
Variedad & - & $\mathrm{C}$ & - & $11.61 \mathrm{~b}$ \\
DMSH & - & & - & 0.46 \\
- & - & T4 & - & $13.8 \mathrm{a}$ \\
- & - & $\mathrm{T} 2$ & - & $12.31 \mathrm{~b}$ \\
Dosis & - & T1 & - & $12.15 \mathrm{~b}$ \\
- & - & $\mathrm{T} 3$ & - & $11.89 \mathrm{~b}$ \\
- & - & T0 & - & $10.79 \mathrm{c}$ \\
DMSH & - & - & 1.05 & - \\
\hline
\end{tabular}

Valores con la misma letra en la misma columna no son estadísticamente diferentes (Tukey, $\alpha=0.05)$. DMSH= diferencia mínima significativa honesta; $\mathrm{CV}=$ coeficiente de variación; $\mathrm{E}=$ Variedad estrella; $\mathrm{C}=\mathrm{Variedad}$ celeste; $\mathrm{T} 0=$ Testigo; $\mathrm{T} 1=$ tratamiento uno; $\mathrm{T} 2=$ tratamiento dos; $\mathrm{T} 3=$ tratamiento tres; $\mathrm{T} 4=$ tratamiento cuatro.

El mayor ingreso neto se obtuvo con $\mathrm{H}-562$ y $160 \mathrm{~kg} \mathrm{~N} \mathrm{ha}^{-1}$ independientemente del uso de Bio. En el trabajo de Díaz et al. (2007) se evaluaron dos dosis de $\mathrm{N}$ (300 kg y $400 \mathrm{~kg}$ ) y los investigadores reportaron que el suministro de $400 \mathrm{~kg}_{\text {de }} \mathrm{N} \mathrm{ha}^{-1}$ promovió un mayor efecto en las variables índice de área foliar (IAF), producción de biomasa y rendimiento de grano.

\section{Elección de la función}

Con los resultados obtenidos en el experimento de producción de maíz blanco y después de probar diferentes formas funcionales como la lineal, la cuadrática, la cúbica, la transcendental, la logarítmica y la Cobb-Douglas, las que presentaron mejor ajuste fueron la lineal y la cuadrática. Esta última muestra que a cierto nivel de uso del insumo variable se empiezan a tener incrementos en producción positivos, pero cada vez de menor magnitud (productos marginales decrecientes).

Se llega a un nivel de uso del insumo variable en el que la última unidad de este recurso no incrementa en nada a la producción (producto marginal cero). En este nivel de uso del insumo fertilizante se alcanza la máxima producción por hectárea. Este polinomio de segundo grado permite calcular el óptimo económico y el óptimo técnico, por esta razón es el que se seleccionó y se presenta enseguida: $Y=9129.9023+4.1646 \mathrm{~F}-0.0011 \mathrm{~F}^{2}-1520.2652 \mathrm{C}, \mathrm{R}^{2}=0.61 ; \mathrm{R}^{2}$ ajustado= $0.53 .(3.25) * * *(-1.86) *(-2.28) * *$.

Donde: Y es el rendimiento de maíz en $\mathrm{kg} \mathrm{ha}^{-1}, \mathrm{~F}$ es la fórmula de fertilizante aplicado en $\mathrm{kg} \mathrm{ha}^{-1}$ y $\mathrm{C}$ es una variable dummy (binaria) de manejo de ceros y unos, donde 0 representa el cultivo que se sembró el año previo, el cual fue maíz y 1 se refiere a que no se cultivó nada en el año anterior (barbecho). Los valores de $\mathrm{t}$ en los paréntesis; ***,** y * denotan significancia estadística al $1 \%$, 
$5 \%$ y $10 \%$, respectivamente. Otras variables que pueden influir en el rendimiento de maíz, tales como la precipitación pluvial, el tipo de suelo, la degradación del suelo, la semilla y el tipo de siembra, el método de cosecha, la tierra, el trabajo, la maquinaria, el uso de herbicidas, entre otras, se suponen constantes en un nivel determinado, durante el ciclo del cultivo, el coeficiente independiente representa el rendimiento atribuible a los factores diferentes a la fórmula.

Para obtener el nivel de fertilizante que genera la máxima ganancia o máximo beneficio se busca la igualdad entre el producto marginal dado por la derivada de la función de producción y la relación de precios: precio del insumo/precio del producto, considerando los precios de mercado, imperantes en la zona, del insumo y del producto $\$ 11.50$ y $\$ 5.00$ por $\mathrm{kg}$, respectivamente, se buscó el valor del factor donde el producto marginal sea igual a $2.3=\$ 11.50 / \$ 5.00$. De tal forma que obteniendo la derivada de la función e igualando a 2.3 , de manera similar como en las ecuaciones (4 y 8), se llega a la siguiente expresión: $\frac{\partial \mathrm{Y}}{\partial \mathrm{f}}=\frac{\mathrm{P}_{\mathrm{F}}}{\mathrm{P}_{\mathrm{Y}}}=4.1646-0.0022 \mathrm{~F}=\frac{11.5}{5}=2.3 ; \mathrm{F}=$ $\frac{-1.8646}{-0.0022}=848 \mathrm{~kg} \mathrm{ha}^{-1}$ es la dosis óptima económica o de máxima ganancia.

En cuanto al valor de F que genera el máximo rendimiento por hectárea, se obtiene igualando la derivada de la función a cero (producto marginal $=0$ ) y despejando posteriormente el valor de $\quad F \quad$ se $\quad \frac{\partial \mathrm{Y}}{\partial \mathrm{f}}=0=4.1646-0.0022 \mathrm{~F}=0, \mathrm{~F}=\frac{-4.1646}{-0.0022}=1893 \mathrm{~kg}$ $\mathrm{ha}^{-1}$ es la dosis óptima técnica o de máximo rendimiento.

Con base en la optimización económica, la cantidad óptima de fertilizante que deben aplicar los productores de maíz para obtener el máximo beneficio económico o ganancia (25 226.02 \$ ha-1) es de $848 \mathrm{~kg} \mathrm{ha}^{-1}$. En virtud de que el óptimo es una variable aleatoria es posible estimar una región confidencial para éste como se muestra en (Castellanos-Pérez et al., 2006). Desde el punto de vista del análisis marginal, al productor de maíz le conviene producir cantidades adicionales de este grano, siempre y cuando el ingreso que obtenga por este producto sea mayor o igual que lo que le cuesta una unidad más de F, necesario para producir estas cantidades.

Es decir, le convendrá producir una mayor cantidad de maíz, si el ingreso marginal (IMg) que obtenga por la venta de este grano es superior o igual que, lo que le cuesta un $\mathrm{kg}$ adicional de $\mathrm{F}$ (costo marginal: $\mathrm{CMg}$ ), con el cual produce esa cantidad de grano. Con base en este criterio económico y al de máxima ganancia, como se observa en el (Cuadro 3), ésta se alcanza cuando el $\operatorname{IMg}(\$ 11.50)$ es igual al CMg $(\$ 11.50)$, lo cual es coherente con la elasticidad de producción $\left(\varepsilon_{\mathrm{p}}=\right.$ $0.19)$, que corresponde a la región económica de la función de producción $\left(0<\varepsilon_{\mathrm{p}}<1\right)$.

Cuadro 3. Variables analizadas: fertilizante, rendimiento, ingreso total, ingreso marginal, costo variable, costo marginal y ganancia o beneficio.

\begin{tabular}{ccccccc}
\hline $\begin{array}{c}\text { Fertilizante } \\
\text { aplicado }\end{array}$ & Rendimiento & $\begin{array}{c}\text { Ingreso } \\
\text { total en } \$\end{array}$ & $\begin{array}{c}\text { Ingreso } \\
\text { marginal en }\end{array}$ & $\begin{array}{c}\text { Costo } \\
\text { variable de }\end{array}$ & $\begin{array}{c}\text { Costo } \\
\text { marginal en }\end{array}$ & $\begin{array}{c}\text { Ganancia o } \\
\text { beneficio }\end{array}$ \\
\hline \multirow{2}{*}{${\left.\mathrm{F}: \mathrm{kg} \mathrm{ha}^{-1}\right)}$} & $\left(\mathrm{Y}: \mathrm{kg} \mathrm{ha}^{-1}\right)$ & \multicolumn{2}{c}{$(\mathrm{IT}=\$(\mathrm{IM}=\mathrm{F} \$(\mathrm{CVF}=\$(\mathrm{CMg}=\mathrm{en} \$(\pi=\mathrm{IT}$} \\
\cline { 2 - 7 } $\mathrm{Pm} * \mathrm{Y})$ & $\Delta \mathrm{IT} / \Delta \mathrm{Y})$ & $\mathrm{Pf} * \mathrm{~F})$ & $\Delta \mathrm{CVF} / \Delta \mathrm{Y})$ & $\mathrm{C})$ \\
\hline 846 & 10346 & 51728.01 & - & 9729 & - & 25226.01 \\
847 & 10348 & 51739.52 & 11.51 & 9740.5 & 11.5 & 25226.02 \\
848 & 10350 & 51751.02 & 11.5 & 9752 & 11.5 & 25226.02 \\
\hline
\end{tabular}




\begin{tabular}{|c|c|c|c|c|c|c|}
\hline $\begin{array}{c}\text { Fertilizante } \\
\text { aplicado }\end{array}$ & Rendimiento & $\begin{array}{l}\text { Ingreso } \\
\text { total en \$ }\end{array}$ & $\begin{array}{l}\text { Ingreso } \\
\text { marginal en }\end{array}$ & $\begin{array}{c}\text { Costo } \\
\text { variable de }\end{array}$ & $\begin{array}{c}\text { Costo } \\
\text { marginal en }\end{array}$ & $\begin{array}{c}\text { Ganancia o } \\
\text { beneficio }\end{array}$ \\
\hline \multirow{2}{*}{$\left(\mathrm{F}: \mathrm{kg} \mathrm{ha}^{-1}\right)$} & \multirow{2}{*}{$\left(\mathrm{Y}: \mathrm{kg} \mathrm{ha}^{-1}\right)$} & \multicolumn{5}{|c|}{$(\mathrm{IT}=\$(\mathrm{IM}=\mathrm{F} \$(\mathrm{CVF}=\$(\mathrm{CMg}=$ en $\$(\pi=\mathrm{IT}$} \\
\hline & & $\mathrm{Pm} * \mathrm{Y})$ & $\Delta \mathrm{IT} / \Delta \mathrm{Y})$ & $\mathrm{Pf} * \mathrm{~F})$ & $\Delta \mathrm{CVF} / \Delta \mathrm{Y})$ & C) \\
\hline 849 & & 51762.51 & 11.49 & 9763.5 & 11.5 & 25226.01 \\
\hline 1892 & & 57757 & 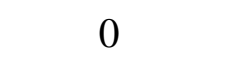 & 21758 & .5 & 5.15 \\
\hline 1893 & 11551 & 57757.15 & 0.01 & 21769.5 & 11.5 & 19214.65 \\
\hline 1894 & 11551 & 57757.15 & -0.01 & 21781 & 11.5 & 19203.15 \\
\hline
\end{tabular}

$\partial \mathrm{Y} / \partial \mathrm{I}$ es la derivada parcial de $\mathrm{Y}$ con respecto a $\mathrm{F}$. $\$=$ peso; $\mathrm{Pm}=$ precio de maíz $=5.00 \$ \mathrm{~kg}^{-1} ; \mathrm{Pf}=$ precio de la fórmula de fertilizante $=1150 \$ \mathrm{~kg}^{-1} ; \mathrm{CVF}=$ costo variable del fertilizante; $\Delta=$ es el cambio o variación; $\mathrm{C}=\mathrm{CVF}+\mathrm{CF}$. Donde: $\mathrm{CF}=$ costo fijo $=\$ 16773.00$.

Por otra parte, la cantidad óptima técnica de F que genera el máximo rendimiento es de $1893 \mathrm{~kg}$ $h^{-1}$, esta cantidad máxima de maíz por hectárea no produce la máxima ganancia, por lo que buscar el mayor rendimiento por hectárea no reditúa, necesariamente, el máximo beneficio económico que buscaría todo productor racional que produce maíz para vender en el mercado de éste. En el caso de productores pequeños, que sólo cuenten con pocos recursos para comprar 424 $\mathrm{kg}$ de $\mathrm{F}$, deben aplicar este nutriente en $1 / 2$ ha y obtendrían una ganancia por peso gastado en $\mathrm{F}$ de $\$ 1.12$. El cual contrasta con el resultado de Aguilar-Carpio et al. (2015), quienes emplearon Bio más $80 \mathrm{~kg} \mathrm{~N} \mathrm{ha}^{-1}$ con $\mathrm{H}-562$ y obtuvieron la mayor ganancia por peso invertido, toda vez que por cada peso recuperaron $\$ 4.33$.

\section{Conclusiones}

De los cuatro tratamientos evaluados, el que tuvo la mejor respuesta en la variable evaluada de rendimiento fue el T4 (la dosis más elevada de fertilización), por lo que en este aspecto fue el mejor. Mientras que para esta misma variable el tratamiento testigo presentó los valores más bajos. Con la Variedad celeste y la dosis más elevada se obtuvo el rendimiento más alto el cual fue de $13.83 \mathrm{t} \mathrm{ha}^{-1}$, mientras que con la Variedad estrella y esta misma dosis de fertilización se registró un rendimiento muy cercano al anterior de $13.77 \mathrm{t} \mathrm{ha}^{-1}$. La función de producción cuadrática de maíz blanco estimada permite obtener la dosis óptima económica o de máxima ganancia de F (848 kg $\left.\mathrm{ha}^{-1}\right)$ y la dosis óptima técnica o de máximo rendimiento $\left(1893 \mathrm{~kg} \mathrm{ha}^{-1}\right)$.

Los resultados obtenidos pueden servir de criterio económico para la toma decisiones de producción de agricultores pequeños de maíz blanco, que cuentan con menos recursos para comprar el fertilizante, por lo que, usando de manera óptima este recurso, podrían aplicar sólo $424 \mathrm{~kg}$ de F en media hectárea y así obtener la máxima ganancia neta por peso gastado en $\mathrm{F}$, como de productores medianos y grandes, que disponen de más recursos para adquirir la cantidad de F que les permita alcanzar la máxima ganancia. Se recomienda que en futuras investigaciones se amplíe la región por explorar, de tal manera que, el tamaño permita observar el tipo de respuesta clásico. También es conveniente explorar un diseño experimental de bloques al azar. 


\section{Literatura citada}

Aguilar-Carpio, C.; Escalante-Estrada, J.; Aguilar-Mariscal, I.; Mejía-Contreras, J. A.; CondeMartínez, V. y Trinidad-Santos, A. 2015. Rendimiento y rentabilidad de maíz en función del genotipo, biofertilizante y nitrógeno, en clima cálido. Trop. Subtrop. Agroecosys. 18(2):151-163.

Beattie, B. R. and Taylor C. R. 1993. The economics of production. Krieger publishing company. Reprint (Ed.). Malabar, Florida, USA. 64-67 pp.

Castellanos-Pérez, M.; Martínez-Garza, A.; Beatriz-Colmenares, C.; Martínez-Damián, M. A. y Rendón-Sánchez, G. 2006. Región confidencial para el óptimo económico de una función de producción Cobb-Douglas. Agrociencia. 40(1):117-124.

CONABIO. 2021. Comisión Nacional para el Conocimiento y Uso de la Biodiversidad. Razas de maíz de México. https://www.biodiversidad.gob.mx/diversidad/alimentos/maices/razasde-maiz.

Díaz, T.; Pérez, N. W.; Páez, F.; López, A. y Partidas, L. 2007. Evaluación del crecimiento del maíz (Zea mays L.) en función de dos técnicas de riego y diferentes niveles de nitrógeno. Rev. Cienc. Técn. Agropec. 16(4):84-87. https://www.redalyc.org/pdf/932/93216418.pdf.

Jauregui, M. A. and Sain, G. E. 1992. Continuous economic analysis of crop response to fertilizer in on-farm research. CIMMYT economics paper no. 3. Mexico, DF. 2-13; 35-46; 53-60 pp.

Kato, Y. T. A.; Mapes, S. C.; Mera, O. L.; Serratos, H. J. A. y Bye, B. R. A. 2009. Origen y clasificación del maíz: una revisión analítica. Impresora Apolo. México, DF. 22-25 pp.

Nicholson, W. 2002. Microeconomics theory: basic principles and extensions. Thomson Learning. Eighth (Ed.). Inc, USA. 215-220 pp.

Martínez, G. A. 1972. Aspectos económicos del diseño y análisis de experimentos. Escuela Nacional de Agricultura. Colegio de Postgraduados. Estado de México. México. 7-35 pp.

Martínez-Gutiérrez, A.; Zamudio-González, B.; Tadeo-Robledo, M.; Espinosa-Calderón, A.; Cardoso-Galvão, J. C.; Vázquez-Carrillo, G. y Turrent-Fernández, A. 2018. Rendimiento de híbridos de maíz grano blanco en cinco localidades de Valles Altos de México. Rev. Mex. Cienc. Agríc. 9(7): 1448-1456.

Portillo, V. M.; Pérez, S. F.; Figueroa, H. E.; Godínez, M. E.; Pérez, S. T. y Barrios, P. G. 2015. La función de producción cúbica, su aplicación en la agricultura. Rev. Mex. Agro. 37(2):7-24.

SAGARPA. 2012. Secretaría de Agricultura, Ganadería, Pesca y Alimentación. Memoria documental del programa 'modernización sustentable de la agricultura tradicional' 20102012. http://observatoriogeograficoamericalatina.org.mx/egal14/Geografiasocioeconomi ca/Geografiaagricola/50.pdf.

SADER-SIAP. 2020. Secretaría de Agricultura y Desarrollo Rural. Sistema de Información Agropecuaria Panorama Agroalimentario 2020. https://www.inforural.com.mx/wpcontent/uploads/2020/11/Atlas-Agroalimentario-2020.pdf.

Wellhausen, E. J.; Roberts, L. M. y Hernández, X. E. 1951. Las razas de maíz en México, su origen y distribución. Imprenta “Aldina”. México, DF. https://www.ars.usda.gov/ARSUserFiles/ 50301000/Races_of_Maize/Raza_Mexico_0_Boo k.pdf.

Yunes, N. A. y López, L. J. 2021. La política agrícola en México: evaluación a partir de una tipología de productores. Estud. Sociol. 39(116):507-511. https://doi.org/10.24201/ es.2020v39n116.2017.

Zamudio, G. B.; Félix, R. A.; Martínez G. A.; Cardoso, G. J. C.; Espinosa, C. A. y Tadeo R. M. 2015. Producción de híbridos de maíz con urea estabilizada y nutrición foliar. Rev. Mex. Cienc. Agríc. 9(6):1231-1241. 